$10,11,05$

\title{
Фазовые переходы в атиферромагнитной модели Гейзенберга на объемно-центрированной кубической решетке с учетом взаимодействий следующих ближайших соседей
}

\author{
(C) А.К. Муртазаев ${ }^{1,2}$, М.К. Рамазанов ${ }^{1}$, Д.Р. Курбанова ${ }^{1, \uparrow}$, М.К. Бадиев ${ }^{1}$ \\ ${ }^{1}$ Институт фризики ДагНЦ РАН, \\ Махачкала, Россия \\ 2 Дагестанский государственный университет, \\ Махачкала, Россия \\ I E-mail: d_kurbanova1990@mail.ru
}

На основе репличного алгоритма метода Монте-Карло и гистограммного анализа данных изучен фазовые переходы в трехмерной антиферромагнитной модели Гейзенберга на объемно-центрированной кубической решетке с учетом взаимодействий следующих ближайших соседей. Исследование проведено для соотношения величин обменного взаимодействия следующих и ближайших соседей $r=1$. Установлено, что для данной модели переход из антиферромагнитной фазы в парамагнитную фазу является фазовым переходом первого рода.

Исследование выполнено при финансовой поддержке РФФИ в рамках научных проектов № 16-02-00214-а и № 18-32-00391 мол_а.

DOI: $10.21883 /$ FTT.2018.06.45993.15M

\section{1. Введение}

В настоящее время фрустрации в магнитных системах являются предметом повышенного внимания, так как могут вызывать разнообразные неустойчивые состояния, подавляя более стандартные упорядоченные состояния. Примерами таких систем являются спиновые жидкости $[1,2]$ и спиновый лед $[3,4]$, в которых магнитные моменты не полностью упорядочиваются даже при низких температурах или неколлениарных магнитных структурах. Последнее состояние может возникнуть в результате взаимодействия спин-орбитальной связи и магнетизма, например, в случае скирмионов [5], вихрей кристаллов [6,7] или посредством конкуренции [8-10] антиферромагнитного обменного взаимодействия и кинетической энергии электронов, которым, как правило свойственен ферромагнитный порядок.

Причины возникновения фрустрации различны, от геометрии решетки по орбитальным симметриям до более общих конкурирующих состояний в Гамильтониане. Мы здесь фокусируем внимание на фрустрации, обусловленной геометрией решетки, в сочетании с конкуренцией обменного взаимодействия.

В данной работе нами предпринята попытка на основе репличного алгоритма метода Монте-Карло (МК) определить род фазового перехода (ФП) трехмерной антиферромагнитной модели Гейзенберга на объемноцентрированной кубической решетке с учетом взаимодействия следующих ближайших соседей.

Теоретические расчеты и численное моделирование методом МК для этой модели были проведены в работах [11-16]. Авторы работ методом МК провели исследование критического поведения модели Гейзенберга на различных типах решеток, вычислили температуру ФП и рассчитали значения термодинамических параметров в критической области. Теоретические исследования, проведенные в работах $[11,12]$, свидетельствуют о том, что для модели Гейзенберга на решетке пирохлора имеет место слабовыраженный ФП первого рода в двух различных типах коллинеарных магнитоупорядоченных состояний. Состояние, реализуемое при более сильной спин-решеточной связи, является кубической симметрией, характеризуемой магнитными Брегговскими пиками, а при более слабой — тетрагональной симметрией, характеризуемой соответственными локальными искажениями.

Интерес к антиферромагнитной модели Гейзенберга на объемно-центрированной кубической решетке обусловлен тем, что учет взаимодействия следующих ближайших соседей может привести к возникновению фрустраций, что усложняет решение задачи. При изучении фрустрированных систем (ФС) до сих пор основное внимание уделялось спиновым системам на квадратной, треугольной и гексагональной решетке [16-29]. Критические свойства ФС на объемно-центрированной кубической решетке с учетом взаимодействий следующих ближайших соседей практически не исследованы.

Из данных, полученных на сегодняшний день, нельзя однозначно определить характер ФП и закономерности изменения критического поведения ФС на объемноцентрированной кубической решетке, и эти вопросы до сих пор остаются открытыми.

Кроме того, первые попытки исследования этой модели предпринимались в то время, когда мощности вычислительных машин и используемые алгоритмы метода МК не позволяли рассчитывать критические параметры с необходимой степенью точности.

Исследование этой модели на основе современных методов и идей позволит получить ответ на ряд вопро- 


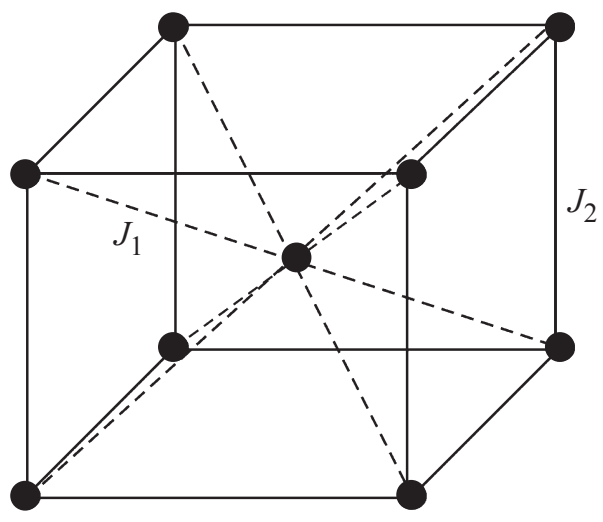

Рис. 1. Схематическое изображение объемно-центрированной кубической решетки.

сов, связанных с природой фрустрированных спиновых систем.

\section{2. Модель и метод исследования}

Антиферромагнитная модель Гейзенберга на объемноцентрированной кубической решетке с учетом взаимодействий ближайших и следующих за ближайшими соседей описывается гамильтонианом:

$$
H=-J_{1} \sum_{\langle i, j\rangle}\left(S_{i} \cdot S_{j}\right)-J_{2} \sum_{\langle i, l\rangle}\left(S_{i} \cdot S_{l}\right),
$$

где $\mathbf{S}_{i}-$ трехкомпонентный единичный вектор $\mathbf{S}_{i}=\left(S_{i}^{x}, S_{i}^{y}, S_{i}^{z}\right)$. Первый член в формуле (1) учитывает обменное взаимодействие ближайших соседей величиной $J_{1}<0$, а второй - следующих за ближайшими соседей $J_{2}<0$. В данной работе рассматривается случай, когда $r=J_{2} / J_{1}=1$, где $r-$ величина взаимодействия следующих ближайших соседей. Схематически эта модель представлена на рис. 1.

Исследование ФП фрустрированных спиновых систем традиционными теоретическими, экспериментальными и численными методами сталкиваются с рядом труднопреодолимых проблем. Это связано с тем, что для таких моделей характерна проблема многочисленных долин локальных минимумов энергии. Строго и последовательно на основе микроскопических гамильтонианов такие системы могут быть изучены методами МК [16-20,22-24,27-30], но обычные методы МК плохо справляются с решением этих проблем. В связи с этим, в последнее время разработано много новых вариантов алгоритмов метода МК, которые позволяют преодолеть эти проблемы. Одними из наиболее мощных и эффективных в исследовании ФП и критических явлений в ФС оказались репличные алгоритмы метода МК [31,32]. Поэтому в данном исследовании был использован высокоэффективный репличный обменный алгоритм метода МК.

Расчеты проводились для систем с периодическими граничными условиями (ПГУ) и линейными размерами $L \times L \times L=N, L=24-60$, где $L$ измеряется в размерах элементарной ячейки. Для вывода системы в состояние термодинамического равновесия отсекался неравновесный участок длиной $\tau_{0}=4 \cdot 10^{5}$ шагов МК на спин, что в несколько раз больше длины неравновесного участка. Усреднение термодинамических параметров проводилось вдоль марковской цепи длиной до $\tau=500 \tau_{0}$ шагов МК на спин.

\section{3. Результаты моделирования}

Для анализа характера ФП, особенностей поведения тепловых характеристик вблизи критической точки и определения критической температуры $T_{N}$ наиболее эффективным является метод кумулянтов Биндера четвертого порядка [21]

$$
\begin{aligned}
& V_{L}=1-\frac{\left\langle U^{4}\right\rangle_{L}}{3\left\langle U^{2}\right\rangle_{L}^{2}}, \\
& U_{L}=1-\frac{\left\langle m^{4}\right\rangle_{L}}{3\left\langle m^{2}\right\rangle_{L}^{2}},
\end{aligned}
$$

где $V_{L}-$ энергетический кумулянт, $U_{L}-$ магнитный кумулянт.

Выражения (2) и (3) позволяют определить критическую температуру $T_{N}$ с большой точностью для ФП первого и второго рода соответственно. Следует отметить, что применение кумулянтов Биндера позволяет также хорошо тестировать тип ФП в системе. Известно, что ФП первого рода характеризуются тем, что величина $V_{L}$ стремится к некоторому нетривиальному значению $V^{*}$ согласно выражению

$$
V_{L}=V^{*}+b L^{-d}
$$

при $L \rightarrow \infty$ и $T=T_{N}(L)$, где величина $V^{*}$ отлична от $2 / 3$, а минимальная величина $U_{L \min }\left(T=T_{\min }\right)$ расходится $U_{L \min }\left(T=T_{\min }\right) \rightarrow-\infty$ при $L \rightarrow \infty$.

В случае ФП второго рода кривые температурной зависимости кумулянтов Биндера UL имеют четко выраженную точку пересечения [21].

На рис. 2 представлена характерная зависимость $U_{L}$ от температуры для разных значений $L$. Из графика видно,

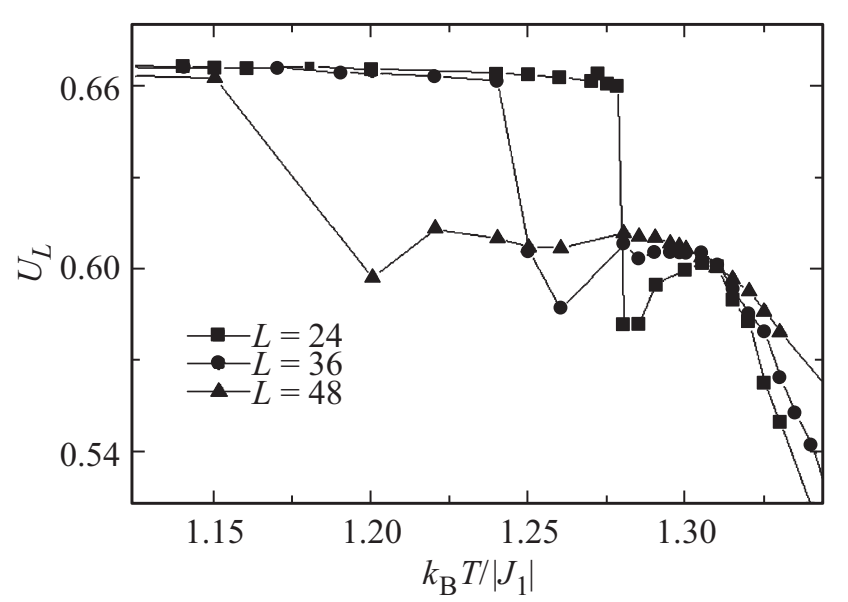

Рис. 2. Зависимость магнитного кумулянта $U_{L}$ от температуры $k_{B} T /\left|J_{1}\right|$. 


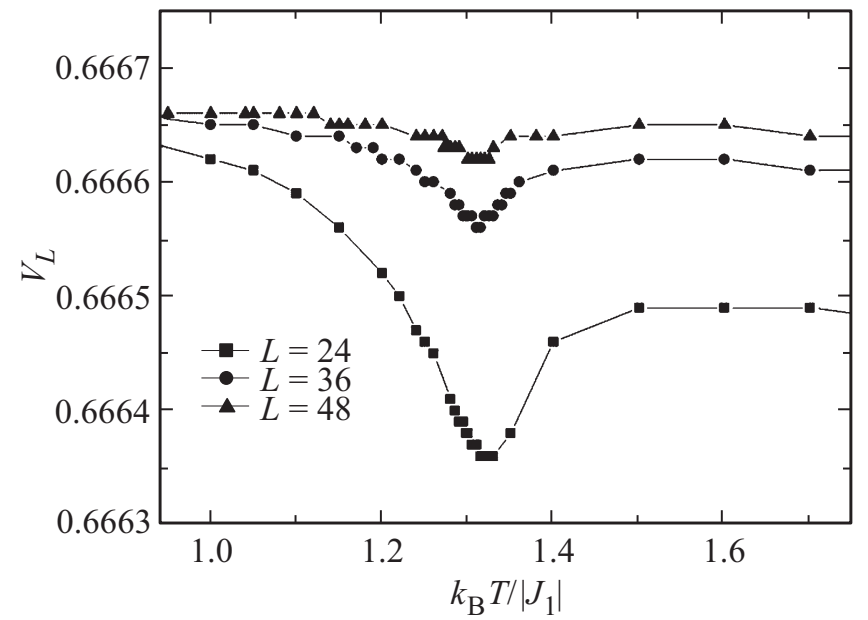

Рис. 3. Зависимость энергетического кумулянта $V_{L}$ от температуры $k_{B} T /\left|J_{1}\right|$.

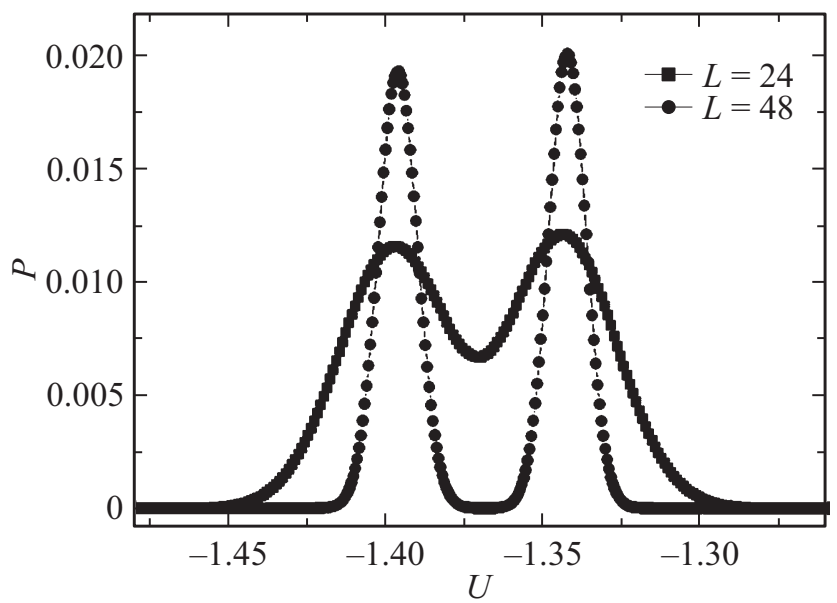

Рис. 4. Гистограмма распределения энергии при $L=24$ и $L=48$.

что в критической области отсутствует четко выраженная точка пересечения, что свидетельствует в пользу наличия в системе фазового перехода первого рода.

На рис. 3 представлена температурная зависимость энергетического кумулянта $V_{L}$ для разных значений $L$. Как видно из графика, величина $V_{L}$ стремится к $2 / 3$, а величина $V^{*}=2 / 3$, что характерно для ФП второго рода. Эта величина рассчитана с помощью выражение (4). Из рис. 3 видно, что для исследуемой модели $V^{*}=0.6666(1)$.

Поэтому для более подробного анализа рода ФП нами использовался гистограммный анализ данных метода МК $[33,34]$. Этот метод позволяет надежно определить род ФП. Методика определения рода ФП этим методом подробно описана в работе [35,36].

Результаты нашей работы, полученные на основе гистограммного анализа, показывают, что фазовый переход из антиферромагнитной фазы в парамагнитную фазу является переходом первого рода. Это продемонстрировано на рис. 4. На этом рисунке представлены гисто- граммы распределения энергии для систем с линейными размерами $L=24$ и $L=48$. Из рисунков видно, что в зависимости вероятности $P(U)$ от энергии $U$ для всех систем наблюдаются два хорошо выраженных максимума, которые свидетельствует в пользу фазового перехода первого рода. Наличие двойного пика на гистограммах распределения энергии является достаточным условием для фазовых переходов первого рода.

\section{4. Заключение}

Исследование фазовых переходов в трехмерной антиферромагнитной модели Гейзенберга на объемноцентрированной кубической решетке с учетом взаимодействия следующих ближайших соседей выполнено с использованием высокоэффективного репличного алгоритма метода Монте-Карло. На основе гистограммного метода и метода кумулянтов Биндера проведен анализ характера фазовых переходов для соотношения величины взаимодействия ближайших и следующих ближайших соседей, равной $r=1$. Показано, что при данном значении $r$ в системе наблюдается фазовый переход первого рода.

\section{Список литературы}

[1] G.A. Fiete, V. Chua, M. Kargarian, R. Lundgren, A. Rüegg, J. Wen, V. Zyuzin. Physica E 44, 845 (2012).

[2] L. Balents. Nature 464, 199 (2010).

[3] S.T. Bramwell. Science 294, 1495 (2001).

[4] Y.-P. Huang, G. Chen, M. Hermele. Phys. Rev. Lett. 112, 167203 (2014).

[5] U.K. Rößler, A.N. Bogdanov, C. Pfleiderer. Nature 442, 797 (2006).

[6] I. Rousochatzakis, U.K. Rössler, J. van den Brink, M. Daghofer. Phys. Rev. B 93, 104417 (2016).

[7] Y. Kamiya, C.D. Batista. Phys. Rev. X 4, 011023 (2014).

[8] I. Martin, C.D. Batista. Phys. Rev. Lett. 101, 156402 (2008).

[9] B.G. Ueland, C.F. Miclea, Y. Kato, O.A. Valenzuela, R.D. McDonald, R. Okazaki, P.H. Tobash, M.A. Torrez, F. Ronning, R. Movshovich, Z. Fisk, E.D. Bauer, I. Martin, J.D. Thompson. Nature Commun. 3, 1067 (2012).

[10] R. Ozawa, S. Hayami, K. Barros, G.-W. Chern, Y. Motome, C.D. Batista. J. Phys. Soc. Jpn. 85, 103703 (2016).

[11] K. Aoyama, H. Kawamura. Phys. Rev. Lett. 116, 257201 (2016).

[12] A. Sadeghi, M. Alaei, F. Shahbazi, M.J.P. Gingras. Phys. Rev. B 91, 140407(R) (2015).

[13] H.T. Diep. J. Science: Adv. Mater. Dev. 1 (2016).

[14] S. Reja, P.S. Anisimov, M. Daghofer. Phys. Rev. B 96, 085144 (2017).

[15] T. Shimokawa, H. Kawamura. J. Phys. Soc. Jpn. 85, 113702 (2016).

[16] А.К. Муртазаев, М.К. Рамазанов, М.К. Бадиев. ФТТ 52, 1557 (2010).

[17] А.К. Муртазаев, М.К. Рамазанов. Письма в ЖЭТФ 106, 72 (2017).

[18] H. Kawamura. J. Phys. Soc. Jap. 61, 1299 (1992). 
[19] A. Mailhot, M.L. Plumer, A. Caille. Phys. Rev. B 50, 6854 (1994).

[20] D.P. Landau, K. Binder. Monte Carlo Simulations in Statistical Physics. Cambridge University Press, Cambridge (2000). P. 384.

[21] Л.Е. Свистов, А.И. Смирнов, Л.А. Прозорова, О.А. Петренко, А.Я. Шапиро, Л.Н. Демьянц. Письма в ЖЭТФ 80, 231 (2004).

[22] А.К. Муртазаев, М.К. Рамазанов, Ф.А. Касан-Оглы, Д.Р. Курбанова. ЖЭТФ 147, 127 (2015).

[23] А.К. Муртазаев, М.К. Рамазанов, Д.Р. Курбанова, М.К. Бадиев, Я.К. Абуев. ФТТ 59, 1082 (2017).

[24] А.К. Муртазаев, М.К. Рамазанов, М.К. Бадиев. ФНТ 37, 1258 (2011).

[25] F.A. Kassan-Ogly, B.N. Filippov, A.K. Murtazaev, M.K. Ramazanov, M.K. Badiev. J. Mag. Mag. Mater. 324, 3418 (2012).

[26] А.К. Муртазаев, М.К. Рамазанов, М.К. Бадиев. ЖЭТФ 142, 338 (2012).

[27] A.K. Murtazaev, M.K. Ramazanov, M.K. Badiev. Physica B: Condens. Matter 476, 1 (2015).

[28] F.A. Kassan-Ogly, A.K. Murtazaev, A.K. Zhuravlev, M.K. Ramazanov, A.I. Proshkin. J. Mag. Mag. Mater. 384, 247 (2015).

[29] M.K. Ramazanov, A.K. Murtazaev, M.A. Magomedov. Solid State Commun. 233, 35 (2016).

[30] А.К. Муртазаев, М.К. Рамазанов. ФТТ 53, 1004 (2011).

[31] A. Mitsutake, Y. Sugita, Y. Okamoto. Biopolymers (Peptide Science) 60, 96 (2001).

[32] А.К. Муртазаев, М.К. Рамазанов, Ф.А. Касан-Оглы, М.К. Бадиев. ЖЭТФ 144, 1239 (2013).

[33] F. Wang, D.P. Landau. Phys. Rev. Lett. 86, 2050 (2001).

[34] F. Wang, D.P. Landau. Phys. Rev. E 64, 056101 (2001).

[35] М.К. Рамазанов. Письма в ЖЭТФ 94, 335 (2011).

[36] М.К. Рамазанов, А.К. Муртазаев. Письма в ЖЭТФ 101, 793 (2015). 